\title{
Predictive value of circulating interleukin- 6 and heart-type fatty acid binding protein for three months clinical outcome in acute cerebral infarction: multiple blood markers profiling study
}

So-Young Park', Jinkwon Kim', Ok-Joon Kim', Jin-Kyeoung Kim², Jihwan Song ${ }^{3}$, Dong-Ah Shin ${ }^{4}$ and Seung-Hun $\mathrm{Oh}^{1 *}$

\begin{abstract}
Introduction: There is no single blood marker for predicting the prognosis in ischemic stroke. A combination of multiple blood markers may enhance the ability to predict long-term outcome following ischemic stroke.

Methods: Blood concentrations of neuronal markers (neuron-specific enolase, visinin-like protein 1, heart type fatty acid binding protein (hFABP) and neuroglobin), astroglial markers (S100B and glial fibrillary acidic protein), inflammatory markers (IL-6, TNF- $\alpha$, and C-reactive protein), blood-brain barrier marker (matrix metalloproteinase 9), and haemostatic markers (D-dimer and PAl-1) were measured within 24 hours after stroke onset. The discrimination and reclassification for favorable and poor outcome were compared after adding individual or a combination of blood markers to the clinical model of stroke outcome.

Results: In multivariate analysis, natural log-transformed (log) IL-6 (odds ratio (OR): 1.75, 95\% Cl: 1.25 to 2.25, $P=$ 0.001 ) and loghFABP (OR: 3.23, 95\% Cl: 1.44 to $7.27, P=0.005$ ) were independently associated with poor outcome. The addition of a single blood marker to the clinical model did not improve the discriminating ability of the clinical model of stroke outcome. However, the addition of the combination of log IL-6 and loghFABP to the clinical model showed improved discrimination (area under receiver operating characteristic (AUROC) curve: 0.939 versus $0.910, P=0.03$ ) and reclassification performance (net reclassification improvement index: $0.18, P=0.005$ ).
\end{abstract}

Conclusions: A combination of circulating IL-6 and hFABP level has an additive clinical value for the prediction of stroke outcome.

\section{Introduction}

The early prediction of long-term clinical outcome following ischemic stroke is important because of the high morbidity and mortality associated with stroke. Intravenous tissue plasminogen activator (tPA) is the only effective treatment for ischemic stroke, but less than $15 \%$ of all patients are eligible for thrombolytic therapy due to its narrow therapeutic window [1]. In patients who were not indicated for thrombolytic therapy, early initiation of treatment to reduce secondary neuronal damage is

\footnotetext{
*Correspondence: ohsh72@chamc.co.kr

'Department of Neurology, CHA Bundang Medical Center, CHA University,

351 Yatap-dong, Seongnam, 463-712, Republic of Korea

Full list of author information is available at the end of the article
}

required for a favorable outcome. Age and initial clinical severity, represented by the National Institutes of Health Stroke Scale (NIHSS) score, are established clinical predictors for long-term outcome [2]. Brain imaging is a powerful diagnostic tool to determine the extent of neuronal injury which is associated with long-term stroke outcome. However, brain imaging has several limitations with regard to feasibility and cost-effectiveness.

Blood biomarkers can be a complementary tool for diagnosis [3], predicting prognosis $[4,5]$ and therapeutic monitoring of novel treatments in ischemic stroke [6]. Blood markers of neuronal injury, astroglial injury, inflammatory mediators, and/or thrombotic/haemostatic proteins are candidate blood markers for early stroke

\section{C) Biomed Central}


recurrence or long-term clinical outcome in previous case-control studies [7-11]. For the clinical application of biomarkers for stroke prognosis, candidate blood markers must satisfy the proposed criteria for an ideal blood marker $[4,12]$; 1) the marker has a statistically independent association with outcome after adjusting for clinical covariates; 2) the marker improves the discriminating ability of the established clinical model; and 3) the marker can reclassify patients at low or high risk for poor outcome across clinically relevant thresholds of predicted probabilities of stroke outcome.

Although many blood markers have been investigated to date, no single blood marker has been proven to be clinically useful to predict stroke outcome [12]. The reason is that most of the previous studies measured a single blood marker in a case-control design with variable time points of sampling. Profiling studies of multiple markers, the mechanisms of which are differentially associated with stroke pathophysiology, have tried to overcome the limitation of a single biomarker for stroke diagnosis [13] or outcome $[4,5,10]$. Several panels of inflammatory and haemostatic markers, such as IL-6, $\mathrm{N}$-terminal pro-brain natriuretic peptide, $\mathrm{C}$-reactive protein (CRP), fibrinogen, and/or D-dimer, have been suggested to be associated with poor clinical outcome and death $[4,5,10]$. However, whether the addition of blood biomarkers improves the predictability of the clinical predictors for stroke outcome remains controversial $[4,5,10]$. Therefore, clinically applicable panels of blood markers should be evaluated in subsequent studies.

The aim of the present study is to measure the blood concentration of multiple blood markers in stroke patients and investigate the clinically available blood markers for prediction of the long-term clinical outcome.

\section{Materials and methods}

\section{Study subjects}

We received informed consent from all patients or their families, and the Institutional Review Board at CHA Bundang Medical Center approved the study protocol (IRB protocol no: 2008-034). Among the 353 patients with clinical presentation of stroke who were admitted to the CHA Bundang Medical Center between January 2009 and June 2010, we included only patients who met the following inclusion criteria: (1) symptom onset between 6 and 24 hours prior to blood sampling, (2) first-ever-stroke without any previous history of neurological disease, (3) adequate access to patient's information, and (4) agreement to participate in the study protocol. We excluded patients with (1) intravenous or intra-arterial thrombolytic therapy $(n=26)$, (2) interventional treatment $(n=6)$, (3) neurological symptoms that resolved within 24 hours $(n=21)$, (4) history of malignancy $(n=11),(5)$ evidence of acute systemic infection (for example, fever, pneumonia, urinary tract infection) at the time of blood sampling $(n=8),(6)$ cerebral hemorrhage and head trauma $(n=14)$, or $(7)$ refusal to participate in the study protocol $(n=83)$. Among the 184 patients, 9 patients were later excluded because the blood samples showed poor quality or hemolysis during preparation and were discarded. Therefore, a total of 175 cases were included in the multiple blood marker study (age, $66 \pm 11$ years, 54\% male).

\section{Assessment of clinical parameters}

Ischemic stroke is defined as a clinically definite stroke in a patient whose brain imaging shows a positive lesion on brain magnetic resonance imaging (MRI) or vascular insufficiency relevant to clinical symptoms. At 72 hours after the stroke, all of the patients underwent diffusion weighed imaging (DWI) of the brain MRI to examine the presence of acute infarction. Additional cerebral angiography (one or combined computed tomography (CT)-, MR and/or conventional cerebral angiography) was performed to detect vascular occlusion in all of the patients during hospitalization. All stroke patients underwent electrocardiography and cardiac enzyme (troponin and $\mathrm{CK}-\mathrm{MB}$ ) evaluation at admission, and none had evidence of concurrent acute coronary syndrome. Additional cardiac tests included trans-thoracic echocardiogram (95\%), trans-esophageal echocardiogram (74\%), and 24-hour electrocardiogram monitoring (77\%).

Vascular risk factors were determined as positive past history, current treatment and positive laboratory findings, including systolic and diastolic blood pressure (SBP and DBP), blood tests for fasting glucose, creatinine, and lipid profile. Hypertension was defined as a high baseline blood pressure (systolic $\geq 140 \mathrm{~mm} \mathrm{Hg}$ or diastolic $\geq$ $90 \mathrm{~mm} \mathrm{Hg}$ ) or a history of antihypertensive medication. Diabetes mellitus (DM) was defined as fasting plasma glucose of $\geq 126 \mathrm{mg} / \mathrm{dL}$ or a history of insulin or oral hypoglycemic therapy. Smoking was defined as current smokers at the time of examination. Hyperlipidemia was defined as fasting serum total cholesterol of $\geq 220 \mathrm{mg} / \mathrm{dL}$ or a history of treatment with a statin. Cardiac disease included a previous history or evidence of valvular heart disease, ischemic heart disease, atrial fibrillation (Afib) and heart failure. All of the stroke patients underwent standardized neurological examination by experienced neurologists during hospitalization. Neurological severity was quantified using NIHSS scores from admission to discharge. Long-term clinical outcome was determined by the use of a modified Rankin scale score at three months (3m-mRS) after symptom onset by the same neurologists who were blinded to laboratory results. The favorable outcome group was defined as patients with $3 \mathrm{~m}$-mRS scores of 0 to $2(n=111)$, and the poor outcome group was defined as patients with $3 \mathrm{~m}$-mRS scores 
$\geq 3(n=64)$. The mechanism of stroke was evaluated according to Trial of Org10172 in Acute Stroke Treatment (TOAST) criteria [14].

\section{Measurement of plasma concentration of biomarkers and infarct volume}

Whole blood (10cc) was drawn on arrival at the emergency department using a tube containing ethylenediaminetetraacetic acid (EDTA) or citrate. The samples were immediately delivered to the laboratory. Plasma and serum were quickly isolated from whole blood via centrifuge (3,000 g for 15 minutes), and the samples were stored at $-80^{\circ} \mathrm{C}$ for later analysis. In laboratories blinded to clinical information, a total of 12 panel markers, including: (1) neuronal markers: neuron-specific enolase (NSE), neuroglobin (Ngb), heart-type fatty acid binding protein (hFABP) and visinin-like protein 1 (VSNL-1); (2) astroglial markers: S100B and glial fibrillary acidic protein (GFAP); (3) inflammatory markers: C-reactive protein (CRP), IL-6 and TNF- $\alpha$; (4) bloodbrain barrier marker: matrix metalloproteinase-9 (MMP9); and (5) haemostatic markers: D-dimer and plasminogen activator inhibitor-1 (PAI-1), were measured using commercially available ELISA kits according to the manufacturer's protocols. We selected blood markers that were plausibly associated with the pathophysiology of ischemic stroke, based on previous experimental and clinical studies. Detailed methods for the measurement of individual blood markers are described in Additional file 1.

Infarct volume was assessed using 72-hour DWI of hyperintense lesions. The infarct size at each slice was initially measured using a semi-computerized, intensitythreshold technique (Image J software, NIH, Bethesda, MA, USA). The infarct volume was calculated as the infarct size multiplied by the inter-slice thickness $(6 \mathrm{~mm})$, and the total infarct volume was determined by summating the infarct volumes at each slice.

\section{Statistical analysis}

The categorical data (sex and presence of vascular risk factors) were compared between two groups using the $\chi 2$ test. Continuous data (age, SBP, DBP, routine laboratory data, and blood markers) were compared between two groups using the Student's t-test or a Mann-Whitney $\mathrm{U}$ test if the variables were not matched to a standard normal distribution. All tested blood markers showed highly skewed data (Kolmogorov-Smirov test $<0.02$ ) and were natural log-transformed ( +1 if zero value is present). As most stroke patients showed negative GFAP (71.4\%) and VSNL-1 (93.1\%) in the peripheral blood at this time point, these markers are regarded as binary variables (positive or negative). Data of continuous variables were expressed as mean \pm standard deviation or median with
IQR. The relationship between blood markers and infarct volume was evaluated with Spearman's correlation coefficient test.

For independent association between individual blood markers and stroke outcome, each natural log-transformed $(\mathrm{log})$ blood marker was entered into a separate model after adjusting for age and initial NIHSS score. We constructed a baseline clinical model including age and initial NIHSS score for stroke outcome using logistic regression analyses [15]. Age and initial NIHSS score showed a strong association with stroke outcome after adjusting for other clinical covariates, such as vascular risk factors, Afib and white blood cell (WBC) count in multivariate analysis $(P<0.01)$. Vascular risk factors, Afib and WBC count were not statistically significant after adjusting for age and initial NIHSS score (all $P>0.05$ ). Although infarct volume and TOAST subtype were associated with stroke outcome, we excluded these variables as clinical covariates because: 1 ) these variables could not be obtained within 24 hours after stroke onset; 2) multi-collinearity was suspected between infarct volume and NIHSS score $(r=0.65, P<0.0001)$; and (3) the statistical power of these variables was weaker than the NIHSS score for prediction of stroke outcome. A $P$ value of $<0.005$ for each blood marker was regarded as significant for multiple comparison hypotheses (comparison of 12 blood markers in this study).

To examine whether the addition of blood markers improved the predictability of the baseline clinical model for stroke outcome, we conducted multivariate logistic regression analysis by entering individual or a combined set of ${ }_{\log }$ IL-6 and $\log _{\text {hFABP into the baseline clinical }}$ model. The changes of -2log likelihood ratio and HosmerLemeshow's model were compared among the models. The ability of the model to discriminate between favorable and poor outcome was assessed by receiver operating characteristic (ROC) curve analysis in each model. The differences in area under the curve (AUC) between the blood marker model and baseline clinical model were tested using the Delong method with regard to whether the addition of individual or combined blood markers improved the discrimination between the favorable and poor outcome groups. Additionally, we calculated the net reclassification improvement (NRI) index and integrated discrimination index (IDI) with the clinically relevant thresholds of the predicted probability of baseline clinical model as proposed by a previous study [4]: 0.10 (very low risk), 0.50 (intermediate risk), and 0.90 (very high risk). We determined these thresholds because we examined whether the addition of blood markers can reclassify the very low-risk and very high-risk groups for poor outcome who need conservative care or aggressive treatment. Values less than 0.05 were considered significant associations. The statistical analyses were performed using SPSS version 18.0 (SPSS, Chicago, IL, USA), MedCalc version 
11.3 (Mariakerke, Belgium), and the $\mathrm{R}$ package for Windows (version 2.12.2).

\section{Results}

The demographic and laboratory characteristics between the favorable and poor outcome groups are described in Table 1. The poor outcome group was older, had a higher WBC count and had a higher prevalence of Afib than the favorable outcome group. The poor outcome group had a higher median initial NIHSS score and larger median DWI volume than the favorable outcome group. The univariate logistic regression model indicates that age (OR: 1.58 (per 10 years), 95\% CI: 1.17 to 2.12, $P=0.002)$, initial NIHSS score (OR: 4.20 (per 3 points), 95\% CI: 2.70 to $6.52, P<0.001$ ), Afib (OR: $4.29,95 \% \mathrm{CI}$ : 2.17 to $8.48, P<0.001$ ) and WBC count (adjusted OR: 1.11 (per $10^{3} / \mu \mathrm{l}$ ), 95\% CI: 1.03 to $1.19, P=0.005$ ) were associated with poor prognosis. TOAST classification, especially small vessel disease subtype and small infarct volume were associated with favorable outcome $(P<0.01)$. In the multivariate analysis of clinical variables, only age and initial NIHSS showed a strong association with poor stroke outcome (all $P<0.01$ ), with the disappearance of significance for Afib and WBC count $(P>0.05)$. Among nine deceased patients $(3 m-m R S=6)$, six died from the stroke itself (three large hemispheric stroke, two basilar stroke, and one cerebral hemorrhage), and three likely died from non-stroke causes (two cardiopulmonary distress, one sepsis).

The median concentrations of individual blood markers are shown in Table 2. The median sampling time after stroke onset was 10 hours (IQR: 7 to 16). The poor outcome group had higher median blood concentrations of hFABP, S100B, IL-6, MMP-9, CRP, and $D$-dimer than the favorable outcome group. The blood concentrations of NSE, TNF- $\alpha$ and PAI- 1 were not different between the two groups. As described above, most of the stroke patients displayed negative GFAP and VSNL-1 in the peripheral blood during this time period, indicating that these proteins are unlikely to be released from the brain to the bloodstream during this time window of stroke.

The logistic regression analysis of individual blood markers after adjusting for age and initial NIHSS score revealed that plasma ${ }_{\log } \mathrm{IL}-6$ (adjusted OR: 1.75, 95\% CI: 1.25 to $2.25, P=0.001$ ) and $\log$ hFABP (adjusted OR: 3.23, $95 \%$ CI: 1.44 to $7.27, P=0.0045)$ reached a statistical significance (Table 3). The statistical significance of

Table 1 Demographic and laboratory characteristics in all stroke, favorable and poor outcome groups

\begin{tabular}{|c|c|c|c|c|}
\hline Characteristic & $\begin{array}{c}\text { All } \\
\text { (number = 175) }\end{array}$ & $\begin{array}{c}\text { Favorable } \\
\text { (number 111) }\end{array}$ & $\begin{array}{c}\text { Poor } \\
\text { (number }=64)\end{array}$ & $P$ \\
\hline Age (years old) & $66 \pm 11$ & $64 \pm 11$ & $69 \pm 10$ & 0.002 \\
\hline Sex (male, \%) & $95(54.3)$ & $56(50.5)$ & $39(60.9)$ & 0.21 \\
\hline Initial NIHSS score & $5(3$ to 10$)$ & $4(2$ to 5$)$ & $12(7$ to 16$)$ & $<0.001^{\mathrm{a}}$ \\
\hline 72 hour-infarct volume $(\mathrm{ml})^{\mathbf{b}}$ & $5.9(1.4$ to 35.0$)$ & $3(1$ to 9$)$ & 55 (5 to 215$)$ & $<0.001^{\mathrm{a}}$ \\
\hline Sampling time (hours) & $10(7$ to 16$)$ & 10 (7 to 17$)$ & $11(8$ to 15$)$ & $0.66^{\mathbf{a}}$ \\
\hline Hypertension (\%) & $101(57.7)$ & $61(55.0)$ & $40(62.5)$ & 0.35 \\
\hline Diabetes mellitus (\%) & $53(30.3)$ & $36(32.4)$ & $17(26.6)$ & 0.50 \\
\hline Hyperlipidemia (\%) & $42(24.0)$ & $26(23.4)$ & $16(25.0)$ & 0.81 \\
\hline Heart failure (\%) & $17(9.7)$ & $8(7.2)$ & $9(14.1)$ & 0.19 \\
\hline Atrial fibrillation (\%) & $53(30.3)$ & $21(18.9)$ & $32(50.0)$ & $<0.001$ \\
\hline Smoking (\%) & $71(40.6)$ & $47(42.3)$ & $24(37.5)$ & 0.63 \\
\hline Previous statin use (\%) & $26(14.9)$ & $17(15.3)$ & $9(14.1)$ & 0.99 \\
\hline Systolic BP (mmHg) & $134 \pm 19$ & $134 \pm 19$ & $133 \pm 18$ & 0.68 \\
\hline Diastolic BP (mmHg) & $82 \pm 12$ & $81 \pm 10$ & $83 \pm 14$ & 0.21 \\
\hline Creatinine $(\mathrm{mg} / \mathrm{dl})$ & $1.07 \pm 0.56$ & $1.04 \pm 0.62$ & $1.11 \pm 0.42$ & 0.46 \\
\hline Fasting glucose (mg/dl) & $138 \pm 55$ & $135 \pm 57$ & $143 \pm 53$ & 0.37 \\
\hline LDL cholesterol (mg/dl) & $115 \pm 37$ & $118 \pm 29$ & $109 \pm 47$ & 0.17 \\
\hline $\operatorname{WBC}\left(10^{3} / \mu \mathrm{l}\right)$ & $7.8 \pm 3.0$ & $7.7 \pm 2.7$ & $9.2 \pm 3.7$ & $<0.01$ \\
\hline TOAST & & & & $<0.01$ \\
\hline LAA & $52(29.7)$ & $35(31.5)$ & $17(26.6)$ & \\
\hline CE & $49(28.0)$ & $25(22.5)$ & $24(37.5)$ & \\
\hline SVD & $38(21.7)$ & $34(30.6)$ & $4(6.3)$ & \\
\hline Others & $36(20.6)$ & $17(15.3)$ & 19 (29.7) & \\
\hline
\end{tabular}

${ }^{a}$ Statistical analysis conducted using the Mann-Whitney $\mathrm{U}$ test; ${ }^{\mathrm{b}}$ hyperintense lesion on diffusion-weighted image 72 hours after stroke. BP, blood pressure; $\mathrm{CE}$, cardioembolism; LAA, large-artery atherosclerosis; LDL, low density lipoprotein cholesterol; NIHSS, National Institutes of Health Stroke Scale; SVD, small vessel disease; TOAST, Trial of Org10172 in the Acute Stroke Treatment; WBC, white blood cell. 
Table 2 Comparison of median concentrations of blood markers between favorable and poor outcome groups

\begin{tabular}{|c|c|c|c|c|}
\hline & $\begin{array}{c}\text { All } \\
\text { (number }=175 \text { ) }\end{array}$ & $\begin{array}{c}\text { Favorable } \\
\text { (number }=111)\end{array}$ & $\begin{array}{c}\text { Poor } \\
\text { (number }=64)\end{array}$ & $P^{a}$ \\
\hline \multicolumn{5}{|l|}{ Neuronal } \\
\hline NSE (ng/ml) & 6.5 (1.9 to 10.8$)$ & $5.6(1.6$ to 10.3$)$ & 7.7 (3.0 to 11.5$)$ & 0.14 \\
\hline $\mathrm{Ngb}(\mathrm{ng} / \mathrm{ml})$ & 0.0 (0.0 to 2.7 ) & 0.0 (0.0 to 0.9 ) & 0.3 (0.0 to 9.7) & $<0.001$ \\
\hline hFABP (ng/ml) & $10.0(6.2$ to 16.3$)$ & $8.4(5.5$ to 11.9$)$ & 16.3 (9.9 to 29.7 ) & $<0.001$ \\
\hline VSNL-1 (\%) ${ }^{\mathbf{b}}$ & $12(6.9)$ & $7(6.3)$ & $5(7.8)$ & 0.95 \\
\hline \multicolumn{5}{|l|}{ Astroglial } \\
\hline S100B (pg/ml) & $31.9(0.0-124.9)$ & 20.0 (0 to 60.3) & 135.2 (40.0 to 344.6) & $<0.001$ \\
\hline GFAP $(\%)^{\mathbf{b}}$ & $50(28.6)$ & $23(20.7)$ & $27(42.2)$ & 0.004 \\
\hline \multicolumn{5}{|l|}{ Inflammatory } \\
\hline IL-6 (pg/ml) & $4.6(0.9$ to 13.0$)$ & 2.2 (0.6 to 5.5 ) & $12.1(6.7$ to 48.0$)$ & $<0.001$ \\
\hline MMP-9 (ng/ml) & 65.4 (30.9 to 124.1$)$ & 45.7 (21.9 to 79.5$)$ & 120.5 (61.8 to 228.3) & $<0.001$ \\
\hline TNF- $\alpha(p g / m l)$ & 2.3 (0.0 to 8.9$)$ & 1.3 (0.0 to 8.0$)$ & 3.3 (0.0 to 10.4) & 0.22 \\
\hline CRP (mg/dl) & 0.26 (0.09 to 0.94$)$ & 0.19 (0.06 to 0.36$)$ & 0.87 (0.18 to 4.40$)$ & $<0.001$ \\
\hline \multicolumn{5}{|l|}{ Haemostatic } \\
\hline PAl-1 (ng/ml) & 11.1 (4.5 to 21.8 ) & $10.3(4.2$ to 23.1$)$ & 11.5 (5.9 to 20.9) & 0.57 \\
\hline D-dimer (ng/ml) & 521 (368 to 1083) & 466 (355 to 933) & 938 (450 to 1629) & 0.001 \\
\hline
\end{tabular}

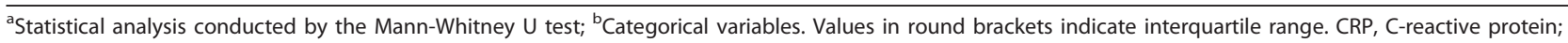
GFAP, glial fibrillary acidic protein; hFABP, heart type fatty acid binding protein; MMP-9 matrix metalloproteinase-9; Ngb, neuroglobin; NSE, neuron-specific enolase; PAI-1, plasminogen activator inhibitor-1; VSNL-1, visinin-like protein 1.

${ }_{\log I L-6}$ and $\log$ hFABP remained after further adjustment for 72-hour infarct volume (natural log-transformed) and Afib (logIL-6, OR: $1.74,95 \%$ CI: 1.24 to $2.44, P=$ 0.003; $\operatorname{loghFABP}$, OR: $3.23,95 \%$ CI: 1.41 to $7.40, P=$ $0.001)$, time of stroke onset $(\log \mathrm{IL}-6$, OR: $1.74,95 \%$ CI: 1.24 to $2.44, P=0.001$; $\operatorname{loghFABP}$, OR: $3.23,95 \%$ CI: 1.41 to $7.40, P=0.005$ ) and previous statin use
( $\log \mathrm{IL}-6, \mathrm{OR}: 1.81,95 \% \mathrm{CI}: 1.27$ to $2.57, P=0.001 ; \log \mathrm{h}-$ FABP, OR: $3.36,95 \%$ CI: 1.46 to $7.47, P=0.004)$. Adding the $\log \mathrm{IL}-6$ and $\log \mathrm{hFABP}$ to the baseline clinical model improved the -2 log likelihood ratio (102.57 versus 123.01, $P=0.01)$ and goodness of fit (Hosmer-Lemeshow's test, $P=0.506)$. No multi-collinearity was found among the tested variables. In the Spearmann's correlation

Table 3 Multivariate logistic regression analysis of blood markers (natural log-transformed) between favorable and poor outcome groups

\begin{tabular}{|c|c|c|c|c|}
\hline & OR $(95 \% \mathrm{Cl})$ & $P$ & Adjusted $\mathrm{OR}^{\mathrm{a}}$ & $P$ \\
\hline \multicolumn{5}{|c|}{ Neuronal markers } \\
\hline NSE & $1.23(0.89-1.68)$ & 0.209 & $0.79(0.49-1.26)$ & 0.325 \\
\hline $\mathrm{Ngb}$ & $1.73(1.28-2.34)$ & 0.0004 & $1.41(0.89-2.24)$ & 0.143 \\
\hline hFABP & $4.90(2.67-9.00)$ & $<0.0001$ & $3.23(1.44-7.27)$ & 0.0045 \\
\hline VSNL-1 ${ }^{\mathbf{b}}$ & $1.26(0.38-4.14)$ & 0.704 & $0.87(0.19-4.02)$ & 0.854 \\
\hline \multicolumn{5}{|c|}{ Astroglial markers } \\
\hline S100B & $1.60(1.39-1.92)$ & $<0.0001$ & $1.20(0.96-1.51)$ & 0.115 \\
\hline GFAP $^{\mathbf{b}}$ & $2.79(1.42-5.49)$ & 0.003 & $1.15(0.41-3.22)$ & 0.791 \\
\hline \multicolumn{5}{|c|}{ Inflammatory markers } \\
\hline $\mathrm{IL}-6$ & $2.23(1.68-2.95)$ & $<0.0001$ & $1.75(1.25-2.25)$ & 0.001 \\
\hline TNF- $\alpha$ & $1.22(0.94-1.57)$ & 0.134 & $1.35(0.88-2.05)$ & 0.166 \\
\hline CRP & $1.63(1.33-1.99)$ & $<0.0001$ & $1.23(0.94-1.62)$ & 0.148 \\
\hline \multicolumn{5}{|c|}{ Blood-brain barrier marker } \\
\hline MMP-9 & $2.19(1.43-3.44)$ & 0.005 & $1.65(1.07-2.07)$ & 0.014 \\
\hline \multicolumn{5}{|c|}{ Haemostatic markers } \\
\hline PAI-1 & $1.05(0.79-1.38)$ & 0.748 & $1.07(0.71-1.61)$ & 0.751 \\
\hline D-dimer & $2.49(1.61-3.86)$ & $<0.0001$ & $1.37(0.74-2.57)$ & 0.319 \\
\hline
\end{tabular}

The favorable group is regarded as the reference (OR: 1.0). ${ }^{a}$ Adjustment of age and initial NIHSS score; ${ }^{b}$ categorical variables. Cl, confidence interval; CRP, Creactive protein; GFAP, glial fibrillary acidic protein; hFABP, heart type fatty acid binding protein; MMP-9 matrix metalloproteinase-9; Ngb, neuroglobin; NSE, neuron-specific enolase; OR, odds ratio; PAl-1, plasminogen activator inhibitor-1; VSNL-1, visinin-like protein 1. 
analysis, infarct volume was significantly correlated with IL-6 $(r=0.38, P<0.001)$ but not hFABP $(r=0.126, P=$ $0.10)$.

In ROC curve analysis, the baseline clinical model showed good discriminating ability (AUC: 0.910) for stroke outcome. The addition of any single marker did not significantly improve the AUC value of the baseline clinical model. Adding a combination of ${ }_{\text {log }} \mathrm{IL}-6$ and $\log$ hFAP to the baseline clinical model (AUC: 0.939, $P<0.03)$ significantly improved the discriminating ability of the baseline clinical model (Table 4 and Figure 1). The addition of other individual markers to the combined model of ${ }_{\log } \mathrm{IL}-6,{ }_{\log }$ hFABP and clinical model did not result in further improvement of AUC in ROC curve analysis ( $P \geq 0.05$ by the DeLong method). We further calculated the in-sample NRI index and IDI of ${ }_{\log } \mathrm{IL}-6$ and ${ }_{\log }$ hFABP in each model (Table 4). Adding ${ }_{\log }$ IL- 6 to the baseline clinical model significantly improved the NRI index $(0.09, P=0.04)$ but adding loghFABP to the baseline clinical model did not improve the NRI index. The addition of both ${ }_{\log } \mathrm{IL}-6$ and ${ }_{\log } \mathrm{h}-$ FABP further enhanced the NRI index of ${ }_{\text {log }} \mathrm{IL}-6(0.18$, $P=0.005)$. The in-sample reclassification table in the model with $\log$ IL- 6 and $\log$ hFABP is presented in Additional file 2. In 64 patients with a poor outcome, seven were reclassified into higher risk categories, and only two were reclassified into lower risk categories. In 111 patients with a favorable outcome, only six were reclassified into higher risk categories, and 17 were reclassified into lower risk categories.

\section{Discussion}

In the present study, we measured multiple blood markers that are plausibly related to the pathophysiology of ischemic stroke to predict functional outcome. The strengths of the present study include the prospective collection of stroke cases, measurement of multiple blood markers in a single study, exact diagnosis of stroke using DWI images in all cases and statistical analysis after adjusting for multiple confounding variables. We found that circulating IL-6 and hFABP levels showed an independent association with stroke prognosis after adjusting for clinical covariates.

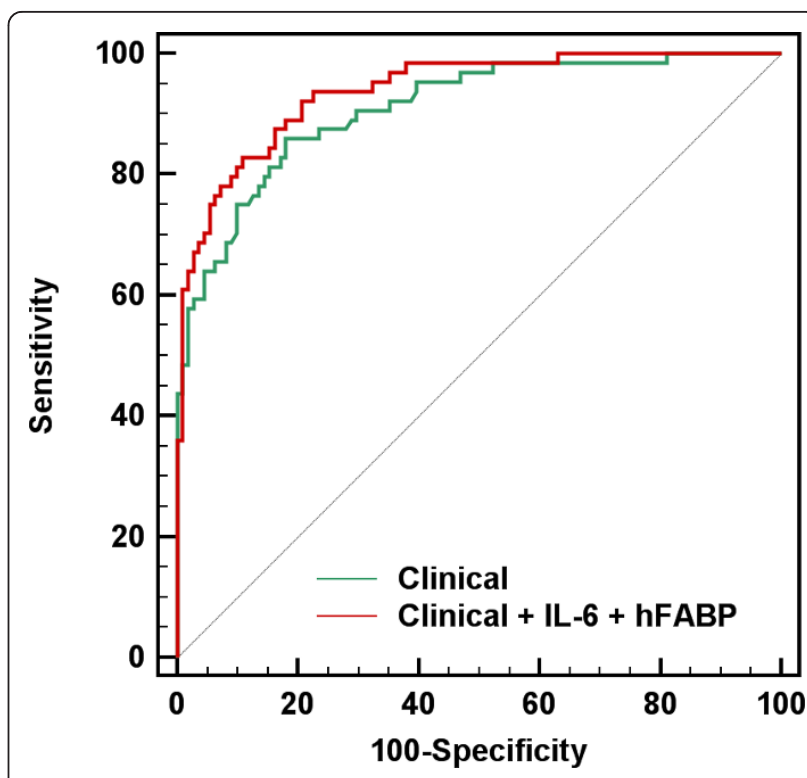

Figure 1 Receiver operating characteristic $(\mathrm{ROC})$ curve of the addition of the combination of ${ }_{\log } \mathrm{IL}-6$ and ${ }_{\log } \mathrm{hFABP}$ to the clinical baseline model.

Moreover, the combination of these two markers showed a significant improvement of discrimination and reclassification for high and low risk of clinical outcome in stroke patients. Other blood markers failed to show a significant clinical value for the prediction of stroke outcome.

Previous studies demonstrated that IL-6 is one of the predictors of poor outcome in stroke [16-20]. Our finding of the strong association between IL- 6 and poor stroke outcome is consistent with those of several studies using multiple blood markers in prospective stroke cohorts $[4,5]$. A systemic inflammatory response develops after brain damage during the acute phase of ischemic stroke. IL-6 is one of the most important mediators of the acute phase response to inflammation. The precise mechanism of the association between elevated IL- 6 levels and poor clinical outcome is unclear. IL-6 up-regulates the expression of adhesion molecules, such as intercellular adhesion molecule-1 (ICAM-1), selectins and integrins on endothelial cells, leukocytes and platelets, during cerebral ischemia,

Table 4 Receiver operating characteristic (ROC) curve and in-sample reclassification analysis of the addition of each or the combination of IL-6 and hFABP (natural log-transformed) to the baseline clinical model for stroke outcome

\begin{tabular}{|c|c|c|c|c|c|c|}
\hline & \multicolumn{2}{|c|}{ Discrimination } & \multicolumn{4}{|c|}{ Reclassification } \\
\hline & AUC (95\% Cl) & $P^{b}$ & NRI & $P^{c}$ & IDI & $P^{d}$ \\
\hline Baseline clinical model ${ }^{\mathbf{a}}$ & $0.910(0.858$ to 0.948$)$ & - & - & - & & \\
\hline$+\log \mid \mathrm{L}-6$ & 0.931 (0.883 to 0.964$)$ & 0.075 & 0.09 & 0.036 & 0.03 & 0.024 \\
\hline$+\log h F A B P$ & $0.926(0.877$ to 0.960$)$ & 0.110 & 0.07 & 0.232 & 0.02 & 0.032 \\
\hline$+\log 1 \mathrm{~L}-6+\log \mathrm{hFABP}$ & 0.939 (0.893 to 0.970$)$ & 0.032 & 0.18 & 0.005 & 0.06 & 0.001 \\
\hline
\end{tabular}

${ }^{a}$ Model with age and initial NIHSS score; ${ }^{b} P$-value for AUC using DeLong method with the baseline clinical model as a reference; ${ }^{c} P$-value for net classification improvement with the baseline clinical model as a reference; ${ }^{d} P$-value for integrated discrimination improvement with the baseline clinical model as a reference. AUC, area under curve; IDI, integrated discrimination improvement; log, natural log-transformed value; NRI, net reclassification improvement. 
which leads to secondary neuronal damage [21]. IL-6 also increases the levels of fibrinogen and von Willebrand factor, resulting in hypercoagulant status [22]. IL-6 expression is increased in the brain following ischemia, and damaged neurons may contribute to increased IL-6 levels [23]. Although the source of circulating IL-6 during the acute phase of stroke is still unknown, the early increase in IL-6 and its correlation with poor outcome support the role of acute inflammation in the pathophysiology of stroke.

Using a blood IL-6, we did not find any improvement in the prognostic performance of the clinical model for stroke outcome despite the close pathophysiological relationship between IL- 6 and cerebral ischemia. Our results are consistent with previous studies which showed that IL-6 lacks clinical usefulness in the discrimination and reclassification between favorable and poor outcome groups of stroke patients $[4,5]$. In our data, the combined use of IL- 6 and hFABP significantly improved the discriminating ability of the clinical model poor stroke outcome. The addition of IL- 6 and hFABP significantly reclassified patients at low- and high-risk for poor outcome across the clinically relevant threshold. hFABP is a small cytoplasmic protein $(15 \mathrm{kDa})$ that is involved in active fatty acid metabolism in the myocardium and neuronal cell bodies in the central nervous system [24]. Plasma hFABP level is known to be a sensitive biomarker of acute coronary ischemia [25,26]. In ischemic stroke, plasma hFABP levels were found to be elevated in several case-control studies [27-29]. Plasma hFABP levels six hours after stroke were associated with early neurological severity (NIHSS score at 10 days) and long-term outcome (mRS at three months) $[28,29]$. The precise mechanism of the association between blood hFABP and stroke outcome is unclear. One plausible explanation is that hFABP is released from the brain following cerebral ischemia. Proteomic analysis showed that hFABP levels in cerebrospinal fluid were elevated in deceased stroke patients [27]. However, we did not find a significant correlation between infarct volume and hFABP, indicating the extracerebral origin of hFABP release following stroke. A second explanation is that the elevation of blood hFABP reflects acute cardiovascular dysfunction associated with neuronal injury [30], which is associated with poor outcome. A third explanation is that hFABP is elevated in other conditions, such as heart failure [31], sepsis [32], pulmonary embolism [33], and metabolic syndrome [34], which may be an underlying substrate for poor stroke outcome. Further studies are required to clarify the origin of hFABP release during the acute phase of stroke and its predictive value for stroke outcome.

Neuro-astroglial markers are candidate stroke biomarkers because they are hypothetically more specific to brain injury than inflammatory and haemostatic markers.
Several case-control studies showed that blood levels of S100B, NSE and GFAP are elevated in acute stroke, and are associated with poor stroke outcome [7-9]. We evaluated the blood profiling of several neuro-astroglial markers, including candidate neuronal markers (VSNL-1 and $\mathrm{Ngb}$ ) that have not been tested in human stroke patients $[35,36]$. None of the tested markers, with the exception of hFABP, reached statistical significance. The lack of an association between blood levels and stroke outcome in the present study is attributable to their slow release kinetics from damaged tissue to peripheral blood (that is, their peak plasma levels were reached at 48 to 72 hours after stroke onset) $[8,28]$.

The limitations of the present study should be addressed. First, we did not perform serial measurements of the blood markers. The inflammatory process and release of neuro-astroglial proteins are prominent for several days after a stroke. Therefore, the measurement of blood markers within 24 hours after stroke onset weakens the potential application. The aim of our study is to search for panel markers within 24 hours of stroke onset for prediction of stroke outcome, as prediction of stroke outcome is required as soon as possible considering the high mortality and morbidity of stroke. We also excluded patients with their onset within six hours because most cases during this time period need thrombolytic therapy, which may confound the clinical outcome of cerebral infarction, and because our experience has shown that most of the tested biomarkers (especially neuro- and astroglial proteins) are not elevated within six hours after symptom onset. Second, it is unknown whether the elevation of blood markers represents a consequence or a pre-morbid status of stroke, as inflammatory and haemostatic markers are elevated in non-cerebral conditions, such as systemic infection, cancer or metabolic diseases. Further studies that involve serial measurement of blood marker levels are required to resolve this issue. Third, the sample size of our study was small. Our results should be interpreted with caution, and external validation is required to be generalized in clinical practice. Fourth, we could not examine the candidate blood markers for prediction of stroke outcome in the previous studies, including brain natriuretic peptide (BNP or NT-proBNP) [4], midregional pro-atrial natriuretic peptide (MR-proANP) [37], lipoprotein-associated phospholipase A2 (LpPlA2) [38], or copeptin [39]. Further studies that expand the panel of blood markers screened are required.

\section{Conclusions}

We found that blood IL- 6 and hFABP levels are associated with poor clinical outcome in patients with ischemic stroke. Single markers did not enhance the discriminating ability of the clinical predictors for stroke outcome. The addition of the IL- 6 and hFABP levels improved the 
prognostic performance of the clinical predictors for stroke outcome. Further studies in a large-scale prospective cohort are required to determine whether these blood proteins are clinically useful in routine clinical practice for prediction of those at high-risk of a poor clinical outcome, who need more intensive therapeutic strategies.

\section{Key messages}

- Combination of multiple blood markers that involve different biological pathways may enhance the predictability of clinical parameters for stroke outcome.

- Blood IL-6 and hFABP levels were independently associated with the functional outcome at three months in patients with ischemic stroke.

- Combination of blood markers including IL-6 and hFABP improved the predictive value of clinical predictors for stroke outcome.

\section{Additional material}

Additional file 1: Methods for individual blood markers

Additional file 2: Reclassification table for favorable and poor stroke outcome.

\begin{abstract}
Abbreviations
3m-mRS: modified Rankin score at three months; Afib: atrial fibrillation; AUC: area under the curve; CRP: C-reactive protein; DBP: diastolic blood pressure; DWI: diffuse weighted image; ELISA: enzyme-linked immunosorbent assay; GFAP: glial fibrillary acidic protein; hFABP: heart-type fatty acid binding protein; IDI: integrated discrimination index; IL-6: interleukin-6; MMP-9: matrix metalloproteinase-9; MRI: magnetic resonance imaging; Ngb: neuroglobin; NIHSS: National Institutes of Health Stroke Scale; NRI: net reclassification improvement; NSE: neuron-specific enolase; OR: odds ratio; PAl-1: plasminogen activator inhibitor-1; ROC: receiver operating characteristic; SBP: systolic blood pressure; TNF-a: tumor necrosis factor-a; TOAST: Trial of Org10172 in Acute Stroke Treatment; VSNL-1: visinin-like portein-1; WBC: white blood cells.
\end{abstract}

\section{Authors' contributions}

SYP, SHO and OJK developed the study concept and design, and wrote the draft. JKK and JS performed the acquisition of data and analysis. JK, DAS and $\mathrm{SHO}$ conducted the interpretation of the data. JS, JKK, DAS and SHO made substantial revision of the report. All authors read and approved the final manuscript for publication.

\section{Competing interests}

The authors declare that they have no competing interests.

\section{Acknowledgements}

We are grateful to Yu-Kyung Kim, Ji-Ye Song, and Ju-Yeon Jeong (Researchers, The Institute for Clinical Research, CHA Bundang Medical Center) for their technical support. This study was supported by a grant of Basic Science Research Program through the National Research Foundation of Korea (NRF) funded by the Ministry of Education, Science and Technology (2011-0021344) and the Korea Healthcare technology R\&D project, Ministry for Health, Welfare \& Family Affairs, Republic of Korea (A111016).

\section{Author details}

'Department of Neurology, CHA Bundang Medical Center, CHA University, 351 Yatap-dong, Seongnam, 463-712, Republic of Korea. ${ }^{2}$ Department of
Pharmacy, CHA University, 222 Yatap-dong, Seongnam, 463-070, Republic of Korea. ${ }^{3}$ Department of Biomedical Science, CHA Stem Cell Institute, CHA University, 606-16 Yeoksam-dong, Seoul, 135-081, Republic of Korea. ${ }^{4}$ Department of Neurosurgery, Yonsei University College of Medicine, 134 Sinchon-dong, Seoul, 120-752, Republic of Korea.

Received: 28 November 2012 Revised: 27 February 2013 Accepted: 13 March 2013 Published: 16 March 2013

\section{References}

1. Katzan IL, Hammer MD, Hixson ED, Furlan AJ, Abou-Chebl A, Nadzam DM: Utilization of intravenous tissue plasminogen activator for acute ischemic stroke. Arch Neurol 2004, 61:346-350.

2. Counsell C, Dennis M, McDowall M: Predicting functional outcome in acute stroke: comparison of a simple six variable model with other predictive systems and informal clinical prediction. I Neurol Neurosurg Psychiatry 2004, 75:401-405.

3. Whiteley W, Wardlaw J, Dennis M, Lowe G, Rumley A, Sattar N, Welsh P, Green A, Andrews M, Graham C, Sandercock P: Blood biomarkers for the diagnosis of acute cerebrovascular diseases: a prospective cohort study. Cerebrovascul Dis 2011, 32:141-147.

4. Whiteley W, Wardlaw J, Dennis M, Lowe G, Rumley A, Sattar N, Welsh P, Green A, Andrews M, Sandercock P: The use of blood biomarkers to predict poor outcome after acute transient ischemic attack or ischemic stroke. Stroke 2012, 43:86-91.

5. Whiteley W, Jackson C, Lewis S, Lowe G, Rumley A, Sandercock P, Wardlaw J, Dennis M, Sudlow C: Inflammatory markers and poor outcome after stroke: a prospective cohort study and systematic review of interleukin-6. PLoS Med 2009, 6:e1000145.

6. Ehrenreich H, Kastner A, Weissenborn K, Streeter J, Sperling S, Wang KK, Worthmann H, Hayes RL, von Ahsen N, Kastrup A, Jeromin A, Herrmann M: Circulating damage marker profiles support a neuroprotective effect of erythropoietin in ischemic stroke patients. Mol Med 2011, 17:1306-1310.

7. Wunderlich MT, Ebert AD, Kratz T, Goertler M, Jost S, Herrmann M: Early neurobehavioral outcome after stroke is related to release of neurobiochemical markers of brain damage. Stroke 1999, 30:1190-1195.

8. Foerch C, Singer OC, Neumann-Haefelin T, du Mesnil de Rochemont R, Steinmetz H, Sitzer M: Evaluation of serum S100B as a surrogate marker for long-term outcome and infarct volume in acute middle cerebral artery infarction. Arch Neurol 2005, 62:1130-1134.

9. Oh SH, Lee JG, Na SJ, Park JH, Choi YC, Kim WJ: Prediction of early clinical severity and extent of neuronal damage in anterior-circulation infarction using the initial serum neuron-specific enolase level. Arch Neurol 2003, 60:37-41.

10. Welsh P, Barber M, Langhorne P, Rumley A, Lowe GD, Stott DJ: Associations of inflammatory and haemostatic biomarkers with poor outcome in acute ischaemic stroke. Cerebrovasc Dis 2009, 27:247-253.

11. Kang DW, Yoo SH, Chun S, Kwon KY, Kwon SU, Koh JY, Kim JS: Inflammatory and hemostatic biomarkers associated with early recurrent ischemic lesions in acute ischemic stroke. Stroke 2009, 40:1653-1658.

12. Whiteley W, Chong WL, Sengupta A, Sandercock P: Blood markers for the prognosis of ischemic stroke: a systematic review. Stroke 2009, 40: e380-389.

13. Laskowitz DT, Kasner SE, Saver J, Remmel KS, Jauch EC: Clinical usefulness of a biomarker-based diagnostic test for acute stroke: the Biomarker Rapid Assessment in Ischemic Injury (BRAIN) study. Stroke 2009, 40:77-85.

14. Adams HP Jr, Bendixen BH, Kappelle LJ, Biller J, Love BB, Gordon DL, Marsh EE: Classification of subtype of acute ischemic stroke. Definitions for use in a multicenter clinical trial. TOAST. Trial of Org 10172 in Acute Stroke Treatment. Stroke 1993, 24:35-41.

15. Konig IR, Ziegler A, Bluhmki E, Hacke W, Bath PM, Sacco RL, Diener HC, Weimar C: Predicting long-term outcome after acute ischemic stroke: a simple index works in patients from controlled clinical trials. Stroke 2008, 39:1821-1826.

16. Blanco M, Castellanos M, Rodriguez-Yanez M, Sobrino T, Leira R, Vivancos J, Lizasoain I, Serena J, Davalos A, Castillo J: High blood pressure and inflammation are associated with poor prognosis in lacunar infarctions. Cerebrovasc Dis 2006, 22:123-129.

17. Rodriguez-Yanez M, Castellanos M, Blanco M, Garcia MM, Nombela F, Serena J, Leira R, Lizasoain I, Davalos A, Castillo J: New-onset hypertension 
and inflammatory response/poor outcome in acute ischemic stroke. Neurology 2006, 67:1973-1978.

18. Shenhar-Tsarfaty S, Ben Assayag E, Bova I, Shopin L, Fried M, Berliner S, Shapira I, Bornstein NM: Interleukin-6 as an early predictor for one-year survival following an ischaemic stroke/transient ischaemic attack. Int J Stroke 2010, 5:16-20.

19. Smith CJ, Emsley HC, Gavin CM, Georgiou RF, Vail A, Barberan EM, del Zoppo GJ, Hallenbeck JM, Rothwell NJ, Hopkins SJ, Tyrrell PJ: Peak plasma interleukin- 6 and other peripheral markers of inflammation in the first week of ischaemic stroke correlate with brain infarct volume, stroke severity and long-term outcome. BMC Neurol 2004, 4:2.

20. Waje-Andreassen U, Krakenes J, Ulvestad E, Thomassen L, Myhr KM, Aarseth J, Vedeler CA: IL-6: an early marker for outcome in acute ischemic stroke. Acta Neurol Scand 2005, 111:360-365.

21. Wang $Q$, Tang XN, Yenari MA: The inflammatory response in stroke. J Neuroimmunol 2007, 184:53-68.

22. Kerr R, Stirling D, Ludlam CA: Interleukin 6 and haemostasis. Br J Haematol 2001, 115:3-12.

23. Suzuki S, Tanaka K, Nogawa S, Nagata E, Ito D, Dembo T, Fukuuchi Y: Temporal profile and cellular localization of interleukin-6 protein after focal cerebral ischemia in rats. J Cereb Blood Flow Metab 1999, 19:1256-1262.

24. Furuhashi M, Hotamisligil GS: Fatty acid-binding proteins: role in metabolic diseases and potential as drug targets. Nat Rev Drug Discov 2008, 7:489-503.

25. Liao J, Chan CP, Cheung YC, Lu JH, Luo Y, Cautherley GW, Glatz JF, Renneberg R: Human heart-type fatty acid-binding protein for on-site diagnosis of early acute myocardial infarction. Int J Cardiol 2009, 133:420-423.

26. Haltern G, Peiniger S, Bufe A, Reiss G, Gulker H, Scheffold T: Comparison of usefulness of heart-type fatty acid binding protein versus cardiac troponin T for diagnosis of acute myocardial infarction. Am J Cardiol 2010, 105:1-9.

27. Zimmermann-Ivol CG, Burkhard PR, Le Floch-Rohr J, Allard L, Hochstrasser DF, Sanchez JC: Fatty acid binding protein as a serum marker for the early diagnosis of stroke: a pilot study. Mol Cell Proteomics 2004, 3:66-72.

28. Wunderlich MT, Hanhoff T, Goertler M, Spener F, Glatz JF, Wallesch CW, Pelsers MM: Release of brain-type and heart-type fatty acid-binding proteins in serum after acute ischaemic stroke. J Neurol 2005, 252:718-724.

29. Park SY, Kim MH, Kim OJ, Ahn HJ, Song JY, Jeong JY, Oh SH: Plasma hearttype fatty acid binding protein level in acute ischemic stroke: Comparative analysis with plasma S100B level for diagnosis of stroke and prediction of long-term clinical outcome. Clin Neurol Neurosurg 2013, 115:405-410

30. Banki NM, Kopelnik A, Dae MW, Miss J, Tung P, Lawton MT, Drew BJ, Foster E, Smith W, Parmley WW, Zaroff JG: Acute neurocardiogenic injury after subarachnoid hemorrhage. Circulation 2005, 112:3314-3319.

31. Ishino M, Takeishi Y, Niizeki T, Watanabe T, Nitobe J, Miyamoto T, Miyashita T, Kitahara T, Suzuki S, Sasaki T, Billim O, Kubota I: Risk stratification of chronic heart failure patients by multiple biomarkers: implications of BNP, H-FABP, and PTX3. Circ J 2008, 72:1800-1805.

32. Jo YH, Kim K, Lee JH, Rhee JE, Lee JH, Kang KW, Rim KP, Hwang SS, Park HM: Heart-type fatty acid-binding protein as a prognostic factor in patients with severe sepsis and septic shock. Am J Emerg Med 2012, 30:1749-1755.

33. Boscheri A, Wunderlich C, Langer M, Schoen S, Wiedemann B, Stolte D, Elmer G, Barthel P, Strasser RH: Correlation of heart-type fatty acidbinding protein with mortality and echocardiographic data in patients with pulmonary embolism at intermediate risk. Am Heart J 2010, 160:294-300.

34. Akbal E, Ozbek M, Gunes F, Akyurek O, Ureten K, Delibasi T: Serum heart type fatty acid binding protein levels in metabolic syndrome. Endocrine 2009, 36:433-437.

35. Laterza OF, Modur VR, Crimmins DL, Olander JV, Landt Y, Lee JM, Ladenson JH: Identification of novel brain biomarkers. Clin Chem 2006, 52:1713-1721.

36. Shang A, Zhou D, Wang L, Gao Y, Fan M, Wang X, Zhou R, Zhang C: Increased neuroglobin levels in the cerebral cortex and serum after ischemia-reperfusion insults. Brain Res 2006, 1078:219-226.
37. Katan M, Fluri F, Schuetz P, Morgenthaler NG, Zweifel C, Bingisser R, Kappos L, Steck A, Engelter ST, Muller B, Christ-Crain M: Midregional proatrial natriuretic peptide and outcome in patients with acute ischemic stroke. J Am Coll Cardiol 2010, 56:1045-1053.

38. Elkind MS, Tai W, Coates K, Paik MC, Sacco RL: High-sensitivity C-reactive protein, lipoprotein-associated phospholipase $\mathrm{A} 2$, and outcome after ischemic stroke. Arch Int Med 2006, 166:2073-2080.

39. Katan M, Fluri F, Morgenthaler NG, Schuetz P, Zweifel C, Bingisser $R$, Muller K, Meckel S, Gass A, Kappos L, Steck AJ, Engelter ST, Muller B, ChristCrain M: Copeptin: a novel, independent prognostic marker in patients with ischemic stroke. Ann Neurol 2009, 66:799-808.

doi:10.1186/cc12564

Cite this article as: Park et al:: Predictive value of circulating interleukin6 and heart-type fatty acid binding protein for three months clinical outcome in acute cerebral infarction: multiple blood markers profiling study. Critical Care 2013 17:R45.

\section{Submit your next manuscript to BioMed Central and take full advantage of:}

- Convenient online submission

- Thorough peer review

- No space constraints or color figure charges

- Immediate publication on acceptance

- Inclusion in PubMed, CAS, Scopus and Google Scholar

- Research which is freely available for redistribution

Submit your manuscript at www.biomedcentral.com/submit
C Biomed Central 